\title{
Topoisomerase I enhances TFIID-TFIIA complex assembly during activation of transcription
}

\author{
Benjamin M. Shykind, ${ }^{1,2}$ Jaesang Kim, ${ }^{1,3}$ Lance Stewart, ${ }^{4}$ James J. Champoux, ${ }^{5}$ and Phillip A. \\ Sharp ${ }^{1,6}$ \\ ${ }^{1}$ Center for Cancer Research and Department of Biology, Massachusetts Institute of Technology, Cambridge, Massachusetts \\ 02139-4307 USA; ${ }^{4}$ Department of Microbiology; and ${ }^{5}$ Department of Biological Structure, School of Medicine, University of \\ Washington, Seattle, Washington 98195 USA
}

The mechanism of coactivation by DNA topoisomerase I (topo I) was examined in a highly defined in vitro transcription system containing Pol II and purified factors. Both stimulation of the basal reaction and coactivation occurred dependent on $\mathrm{TAF}_{\mathrm{II}} \mathrm{s}$. Activation was first observed at the TFIID-TFIIA stage of initiation and maximal activation required the concomitant presence of TFIID, TFIIA, topo I, and activator. Electrophoretic mobility shift assay demonstrated a dramatic enhancement in the formation of the TFIID-TFIIA complex by topo I and activator, dependent on the $\mathrm{TAF}_{\mathrm{II}} \mathrm{s}$. DNase I footprinting confirmed this recruitment. A catalytically inactive topo I, which coactivated transcription, similarly stimulated the rapid formation of the TFIID-TFIIA complex in the presence of activator. A camptothecin-mediated DNA cleavage assay demonstrated the recruitment of topo I to the template by TFIID. Topo I likely functions during activation by enhancing the formation of an active TFIID-TFIIA complex on the promoter.

[Key Words: Topoisomerase I; transcriptional activation; coactivator]

Received August 29, 1996; revised version accepted December 18, 1996.

Initiation of transcription by RNA polymerase II (Pol II) is a multistep process involving six defined activities termed the general transcription factors (GTFs) and Pol II, which assemble on the promoter in an ordered fashion (Buratowski 1994). This assembly is nucleated by TFIID (Samuels et al. 1982; Davison et al. 1983), which binds the TATA box and protects the promoter region from DNase I cleavage from approximately -45 to +40 relative to the start of initiation (Sawadogo and Roeder 1985; Zhou et al. 1992). The TATA-binding protein (TBP) subunit of TFIID contacts a bent conformation of DNA in the minor groove at the TATA box (Nikolov et al. 1992). The remaining subunits of TFIID are termed $\mathrm{TAF}_{\mathrm{II}} \mathrm{S}$ (TBP-associated factors-Pol II) (Dynlacht et al. 1991) and are responsible for the extended footprint of TFIID on the promoter (Verrijzer et al. 1994).

The inability of sequence-specific transcriptional activators to increase the rate of initiation when added to purified reactions in vitro (Meisterernst et al. 1991), and the ability of TBP to support the basal reaction but not activation (Dynlacht et al. 1991), indicated the existence

Present addresses: ${ }^{2}$ Department of Neurobiology and Behavior, Columbia College of Physicians and Surgeons and Howard Hughes Medical Institute, New York, New York 10032 USA; ${ }^{3}$ Division of Biology, California Institute of Technology, Pasadena, California 91125 USA.

${ }^{6}$ Corresponding author.

E-MAIL sharppa@mit.edu; FAX (617) 253-3867. of coactivating factors that are necessary for activation but not required for the basal reaction. These coactivators fall broadly into two categories: $(1)$ members of the $\mathrm{TAF}_{\mathrm{II}} \mathrm{S}$, which are tightly associated with the TBP; and (2) soluble factors not complexed with GTFs. The soluble coactivators defined by biochemistry to date include PC-4 (Ge and Roeder 1994; Kretzschmar et al. 1994), topoisomerase I (topo I) (Kretzschmar et al. 1993; Merino et al. 1993), HMG-1/2 (Shykind et al. 1995), and HMG-17 (Paranjape et al. 1995). The factor TFIIA is not essential, but generally stimulatory, for the basal reaction in purified systems. TFIIA is essential for activation both in nuclear extracts and in highly defined systems (Ma et al. 1993; Ozer et al. 1994; Kobayashi et al. 1995; Shykind et al. 1995), and it is important for activation in vivo (Sun et al. 1994). In yeast, a large complex containing Pol II, SRB proteins, and many other polypeptides can mediate activation (Kim et al. 1994; Koleske and Young 1994). This holoenzyme complex contains coactivating activities tightly associated with the polymerase that are thought to potentiate functional interactions with activators.

Potentiation of transcription could be the result of an increase in the rate of recruitment of a particular factor, or it may come about by facilitating an essential conformational change in a complex. Functional studies in vitro have defined the TFIIB-binding step as a target of 
activator function (Lin and Green 1991), with a proposed conformational change in the factor (Roberts and Green 1994). The TFIID-TFIIA-binding stage has also been shown to be altered during activation (Wang et al. 1992; White et al. 1992; Lieberman and Berk 1994), with a suggestion of a conformational change (Shykind et al. 1995). Functional and physical interactions among activators, coactivators, and basal factors have also been defined. Different activation domains have been proposed to interact functionally with specific $\mathrm{TAF}_{\mathrm{II}} \mathrm{s}$ of the TFIID complex (Goodrich et al. 1993; Hoey et al. 1993; Gill et al. 1994; Tjian and Maniatis 1994). Additionally, multiple activator- $\mathrm{TAF}_{\mathrm{II}}$ interactions have been shown to generate synergy in activation (Sauer et al. 1995). Activation domains have been shown to associate with TBP, TFIIB, TFIIF, TFIIA, and TFIIH. However, these interactions have not been mechanistically integrated into a process accounting for activation in vivo.

Topo I was originally proposed to function in transcription by relieving the torsional stress that accompanies elongation (for review, see Wang 1985) Subsequently, topo I was found to be associated with actively transcribed regions of chromatin (Weisbrod 1982; Fleischmann et al. 1984; Gilmour et al. 1986). Inhibitor studies with drugs such as camptothecin have specifically trapped topo I covalently bound to actively transcribed genes (Stewart and Schutz 1987; Zhang et al. 1988; Bendixen et al. 1990; Stewart et al. 1990). Furthermore, antibodies to topo I interfere with elongation in vivo (Egyhazi and Durban 1987). Recently, topo I was identified biochemically as a coactivator of transcription (Kretzschmar et al. 1993; Merino et al. 1993). Such coactivation probably involves a pre-elongation step as a catalytically inactive mutant of the enzyme is also active in potentiation of activation. Furthermore, topo I was shown to coimmunoprecipitate with the TFIID complex and can bind to TBP (Merino et al. 1993). Thus, this abundant nuclear enzyme may function at two stages of transcription: initiation and elongation.

We have studied the mechanism of stimulation of transcription by the coactivator DNA topo I. In a highly defined in vitro system, topo I exerts its effect at the TFIID-TFIIA binding step. The activation step is accompanied by a dramatic increase in the rate of formation of the TFIID-TFIIA complex with activator and coactivator. TFIIA and $\mathrm{TAF}_{\mathrm{II}} \mathrm{s}$ are required both for transcriptional activation and for enhancement of initiation complex formation. Camptothecin-mediated cleavage of the template shows that TFIID can recruit topo I to the promoter.

\section{Results \\ DNA topo I coactivates transcription in highly purified Pol II system}

Human DNA topo I, purified to homogeneity (see Materials and Methods), was titrated into reactions of highly defined factors containing recombinantly expressed and purified TFIIB, TFIIE, TFIIF, immunoaffinity-purified
TFIID, TFIIA purified to homogeneity (including purification on $\mathrm{Ni}^{++}$columns as the last chromatographic step), highly purifed TFIIH, RNA Pol II, and supercoiled templates. This system is unable to support significant levels of activation in the absence of coactivators (Shykind et al. 1995). In the absence of activator, topo I increased and then repressed basal transcription from the supercoiled templates (Fig. 1A, lanes 1-6). This enhancement of the basal reaction contrasts with previous results where topo I functioned solely as a repressor in the basal reaction (Kretzschmar et al. 1993; Merino et al. 1993). It is possible that stimulation at low concentrations of topo I was not detected in the previous studies because of the failure to titrate in this range. More importantly, in the presence of the activator Gal4-VP16

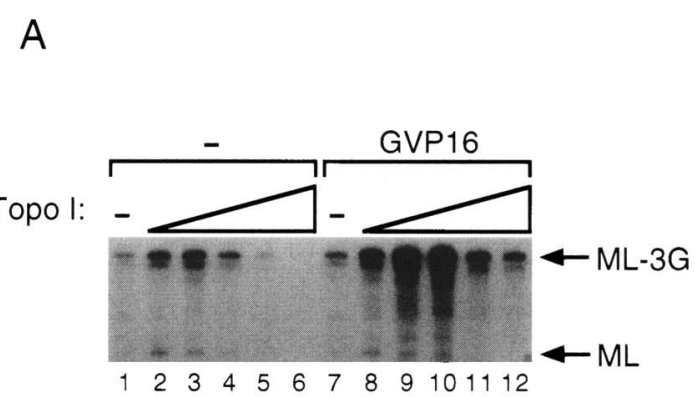

B

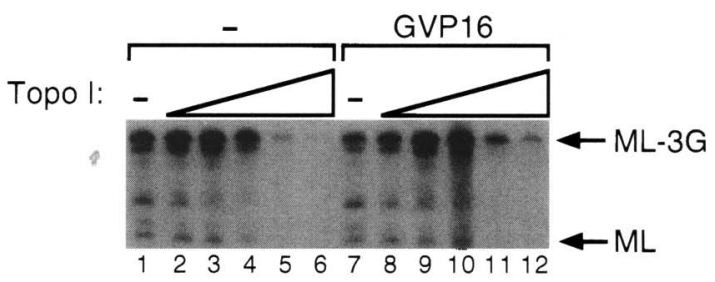

Figure 1. (A) Topo I potentiates activation by Gal4-VP16 on supercoiled major late promoters (MLP). ML transcript (180 nucleotides) was generated by $25 \mathrm{ng}$ of supercoiled MLP basal control promoter. ML-3G transcript (390 nucleotides) was generated by $25 \mathrm{ng}$ of supercoiled MLP with three Gal4-binding sites fused upstream. (Lanes 1-6) Titration of topo I over one log (with $200 \mathrm{ng}$ of topo I maximum) in the absence of activator; (lanes 7-12) titration of topo I over one log in the presence of 10 ng of the synthetic activator Gal4-VP16. Transcription reactions were analyzed, after purification, on $10 \%$ polyacrylamide gels with $8 \mathrm{M}$ urea, and gels were subjected to autoradiography. (B) Topo I does not obviate the requirement for $\mathrm{TAF}_{\mathrm{II}} \mathrm{s}$ in activation by Gal4-VP16. ML transcript (180 nucleotides) was generated by $25 \mathrm{ng}$ supercoiled MLP basal control promoter. ML-3G transcript (390 nucleotides) was generated by $25 \mathrm{ng}$ of supercoiled MLP with three Gal4-binding sites fused upstream. (Lanes 1-6) Titration of topo I over one log (with $200 \mathrm{ng}$ topo I maximum) in the absence of activator. (Lanes 7-12) Titration of topo I over one log in the presence of the synthetic activator Gal4-VP16. Reactions contained $10 \mathrm{ng}$ of recombinant human TBP. Transcription reactions were analyzed, after purification, on $10 \%$ polyacrylamide gels with $8 \mathrm{M}$ urea, and subjected to autoradiography. 
(Fig. 1A, lanes 7-12), topo I potently coactivated transcription from the major late promoter fused to three Gal4-binding sites (ML-3G). The degree of activation with topo I was approximately sixfold over that generated by the addition of activator alone (Fig. 1A, cf. lanes 1,7 , and 10 ) as calculated by comparison of the signals from the ML-3G transcript and the internal control ML transcript. Activation was observed at all topo I concentrations, and the degree increased at levels of topo I that suppressed basal transcription (Fig. 1A, cf. lanes 1, 6, 7, and 12). The ability of topo I to boost the basal reaction is typical of other coactivators such as PC-4 and high mobility group 2 (HMG-2) in this transcription system (Shykind et al. 1995; data not shown).

The role of $\mathrm{TAF}_{\mathrm{II}} \mathrm{s}$ in topo I activity was tested by addition of recombinant TBP in place of holoTFIID. In contrast to the reaction with TFIID, no stimulation of basal transcription occurred upon titration of topo I in reactions containing TBP (Fig. 1B, lanes 1-6). However, repression of transcription was observed at the highest levels of topo I. Furthermore, in agreement with Kretzschmar et al. (1993), Gal4-VP16 did not stimulate transcription under these conditions (Fig. 1B, lanes 7-12). Thus, $\mathrm{TAF}_{\mathrm{II}} \mathrm{s}$ are required for activated transcription in the presence of topo $I$.

\section{Defining the stage of activation}

Coactivation by topo I was examined by order-of-addition experiments. Topo I and activator were added at distinct points during the formation of the initiation complex to determine which stage of initiation was first altered in this process (Fig. 2A). Complexes were formed with the indicated basal factors on templates, in the absence or presence of activator and coactivator, in the first incubation step, and then were followed at the beginning of the second incubation step with the remaining factors and nucleoside triphosphates. Stimulation of transcription was higher when activator and coactivator were added to the template in the second incubation along with the GTFs, than when preincubated with the template (Fig. 2A, lanes 1-3). No difference in activation was observed when TFIID was preincubated with template either alone or in the presence of topo I and Gal4-VP16 (lanes 5,6 ). However, activation was more pronounced when TFIID, TFIIA, activator, and coactivator were coincubated (lane 8) than when the TFIID-TFIIA-binding step was followed by addition of activator and coactivator (lane 9). Generation of a complex of TFIID-TFIIADNA by preincubation that does not subsequently respond to activator and coactivator has been described previously (Wang et al. 1992). The subsequent stages of complex assembly showed a similar requirement for the presence of activator and coactivator during their formation to achieve activation. At the TFIID/TFIIB/TFIIF/ Pol II complex stage (lanes 16-18), very little transcription was observed when activator and coactivator were added in the second incubation, after this complex had formed (cf. lanes 16 and 18). Thus, the TFIID-TFIIA-

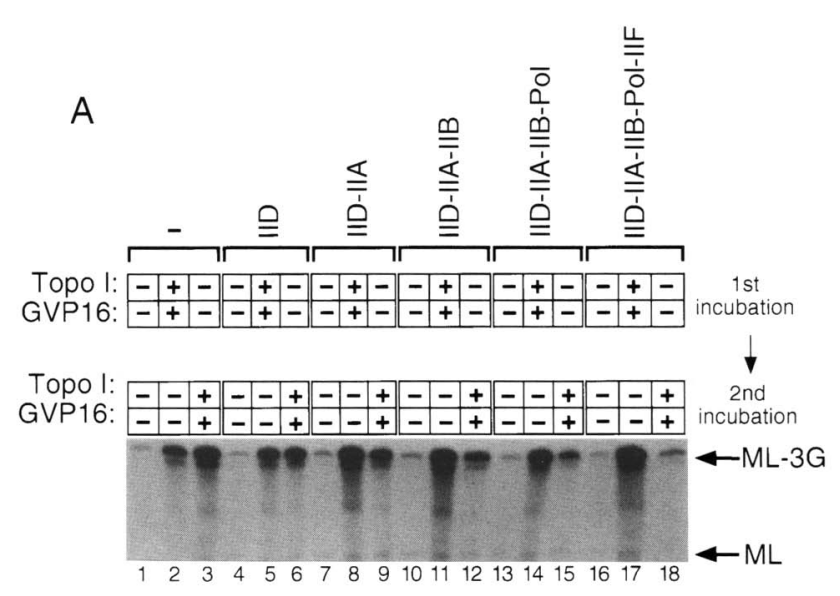

B

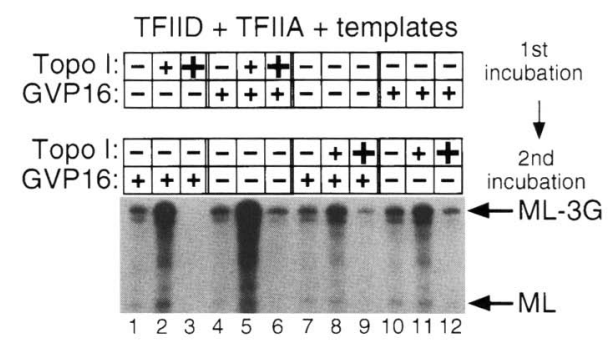

Figure 2. $(A)$ Order-of-addition transcription defines the TFIID-TFIIA step of initiation as critical in activation potentiated by topo I. Gal4-VP16 (10 ng) and topo I (120 ng) were added together either in the first 60 -min incubation step, during the second $60-\mathrm{min}$ incubation step at the different stages of initiation complex formation indicated, or were omitted. ML transcript (180 nucleotides) was generated by $25 \mathrm{ng}$ of supercoiled MLP basal control promoter. ML-3G transcript 1390 nucleotides) was generated by $25 \mathrm{ng}$ of supercoiled MLP with three Gal4-binding sites fused upstream. Transcription reactions were analyzed, after purification, on $10 \%$ polyacrylamide gels with $8 \mathrm{M}$ urea, and subjected to autoradiography. $(B)$ Maximal activation requires the presence of activator and coactivator during TFIID-TFIIA complex formation. Order-of-addition transcription performed as in A. Gal4-VP16 and two concentrations of topo I were added before or after formation of the TFIIDTFIIA complex. NTPs and remaining GTFs were added in the second 60-min incubation. ML transcript (180 nucleotides) was generated by $25 \mathrm{ng}$ of supercoiled MLP basal control promoter. ML-3G transcript (390 nucleotides) was generated by $25 \mathrm{ng}$ of supercoiled MLP with three Gal4-binding sites fused upstream. Transcription reactions were analyzed, after purification, on $10 \%$ polyacrylamide gels with $8 \mathrm{~m}$ urea, and subjected to autoradiography.

binding step is the first stage of initiation altered by topo I during activation.

Formation of the TFIID-TFIIA complex during activation was examined further by an order-of-addition experiment to determine the effect on stimulation of separating the addition of activator and coactivator. In Figure $2 B$, TFIID and TFIIA were incubated with template during the first incubation. Topo I was titrated into the 
reaction either during the first or second incubation; activator was similarly added before or after the TFIIDTFIIA binding step. Maximal activation was only observed when TFIID, TFIIA, topo I, and Gal4-VP16 were concomitantly incubated with template (lanes 4-6). Less activation was observed when the addition of coactivator and activator was separated: Topo I included in the first incubation and activator added in the second incubation (lanes 1-3). When activator was included in the first incubation and topo I in the second (lanes 10-12), or when activator and coactivator were added after the TFIDTFIIA-binding step (lanes 7-9), little activation occurred. Thus, activator and coactivator must both be present at the TFIID-TFIIA-binding stage for maximal activation.

\section{Formation of the TFID-TFIIA complex is facilitated by topo $I$}

The critical nature of the TFIID-TFIIA-binding step in topo I-coactivated stimulation suggested that this complex might form more rapidly in the presence of activator and coactivator. HoloTFIID bound to the TATA box has been characterized previously by electrophoretic mobility shift assay (EMSA) in agarose gels (Zhou et al. 1992). The effects of topo I on formation of TFIID and TFIDTFIIA complexes was examined, in the presence and absence of Gal4-VP16, on a ${ }^{32}$ P-labeled adeno-major late promoter (MLP) probe with three Gal4-binding sites (Fig. 3). Topo I did not alter the binding of saturating amounts of Gal4-VP16 (lanes 1-3), nor did it alone generate a detectable complex with the probe except at much higher concentrations (data not shown). Incubation of TFIID alone formed nearly undetectable amounts of complex, and addition of topo I resulted in a very slight increase (lanes 4-6). Addition of activator did not increase the

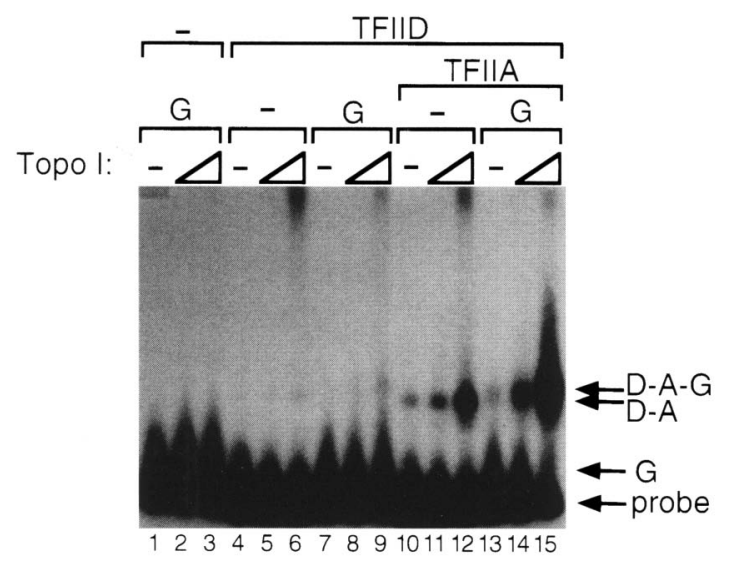

Figure 3. Activator enhances formation of the TFIID-TFIIA complex in the presence of topo I. Agarose $(1.5 \%)$ gel shifts performed with ${ }^{32} \mathrm{P}$-labeled ML probe with three Gal4-binding sites in the presence of $100 \mathrm{ng}$ of competitor poly(dGdC). Topo I was titrated $(0,100,300 \mathrm{ng})$ in the presence of combinations of Gal4-VP16 (G), TFIIA, and holoTFIID. Reactions were incubated for $1 \mathrm{hr}$ at $30^{\circ} \mathrm{C}$ and then analyzed by agarose EMSA. Gels were dried down and subjected to autoradiography.

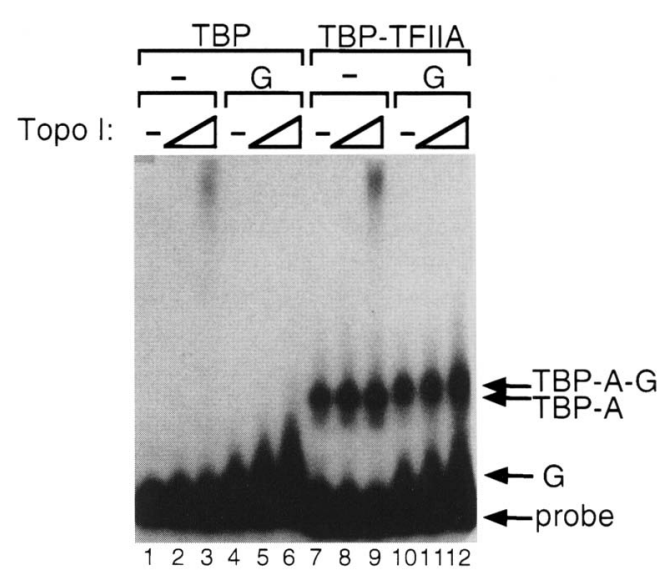

Figure 4. $\mathrm{TAF}_{\mathrm{II}} \mathrm{s}$ are required for enhanced formation of TFIIDTFIIA complex formation. Agarose $(1.5 \%)$ gel shifts performed with ${ }^{32} \mathrm{P}$-labeled ML probe with three Gal4-binding sites in the presence of $100 \mathrm{ng}$ of competitor poly(dGdC). Topo I was titrated $(0,100,300 \mathrm{ng})$ into binding reaction with $10 \mathrm{ng}$ of TBP in the absence (lanes 1-3) and the presence (lanes 4-6) of Gal4VP16 $(G)$, and with TBP and TFIIA in the absence (lanes 7-9) and presence (lanes 10-12) of Gal4-VP16. Reactions were incubated for $1 \mathrm{hr}$ at $30^{\circ} \mathrm{C}$ and then analyzed by agarose-EMSA. Gels were dried down and subjected to autoradiography.

amount of TFIID complex either in the absence (lane 7) or in the presence (lanes 8, 9) of increasing amounts of topoisomerase I. However, formation of a TFIID-TFIIA complex was stimulated by increasing amounts of topo I (lanes 10-12). The presence of activator did not increase the formation of the TFIID-TFIIA complex in the absence of topo I (lane 13). However, in the presence of topo I, activator strongly stimulated the formation of this complex (lanes 14-15). Thus formation of the TFIIDTFIIA complex with topo I and activator, identified as the first critical stage in activation, is correlated with an increase in complex formation.

To determine whether $\mathrm{TAF}_{\mathrm{II}} \mathrm{S}$ were required for the augmentation of complex formation on the TATA box, recombinant human TBP was substituted for holoTFIID. No complex was observed when TBP was incubated with the probe alone either in the absence (lane 1) or in the presence (lanes 2,3) of increasing amounts of topo I (Fig. 4). Furthermore, no complex was observed when activator was included in the absence or presence of topo I (lanes 4-6). A TBP-TFIIA complex readily formed on the probe (lane 7). Interestingly, the amount of complex was not altered by the presence of topo I (lanes 8,9 ), and furthermore, addition of the activator did not alter formation of this complex either in the absence (lane 10) or in the presence (lanes 11,12 ) of topo I. Thus, $\mathrm{TAF}_{\mathrm{II}} \mathrm{s}$ are required for the ability of topo I to stimulate both the formation of the TFIID-TFIIA complex and for the strong stimulation of this complex in the presence of activator.

\section{Rate of complex formation}

The rate of formation of the TFIID-TFIIA complex with 
activator and topo I was examined (Fig. 5). TFIID, TFIIA, and topo I were mixed together on ice and subsequently added to a reaction containing probe, in the presence and absence of activator, and incubated at $30^{\circ} \mathrm{C}$ for increasing amounts of time prior to EMSA. In the absence of activator (lanes 1-5), very little complex was detected after 1 min of incubation (lane 1), but some accumulated after $60 \mathrm{~min}$ of incubation (lane 5). Strikingly, the addition of Gal4-VP16 (lanes 6-10) increased the rate of formation of the complex, such that the full level of complex formation had been reached after 1 min of incubation (lane 6). Thus, the rate of formation of the TFIID-TFIIA complex is increased dramatically by the presence of topo I and activator.

\section{TFIID, TFIIA, topo I, and activator required concomitantly}

Order-of-addition experiments showed that activation mediated by topo I was first detected at the TFIIDTFIIA-binding stage (Fig. 2A) and that maximal stimulation required concomitant incubation of TFIID, TFIIA, activator, and topo I with the template (Fig. 2B). An order-of-addition EMSA was also used to determine the temporal order of factor addition critical for enhanced assembly of the TFIID-TFIIA complex in the presence of topo I and activator (Fig. 6). Various combinations of TFIID, TFIIA, topo I, and activator were added to probe during the first $10-\mathrm{min}$ incubation or were added during the second 5-min incubation; the reactions were subsequently analyzed by EMSA. Incubation of TFIID in the first step without (lanes 1-3) or with (lanes 4-6) TFIIA produced little complex when topo I was added during the second incubation, without respect to the presence of activator or the subsequent addition of TFIIA. TFIID coincubated with topo I and probe (lanes 7-9) similarly

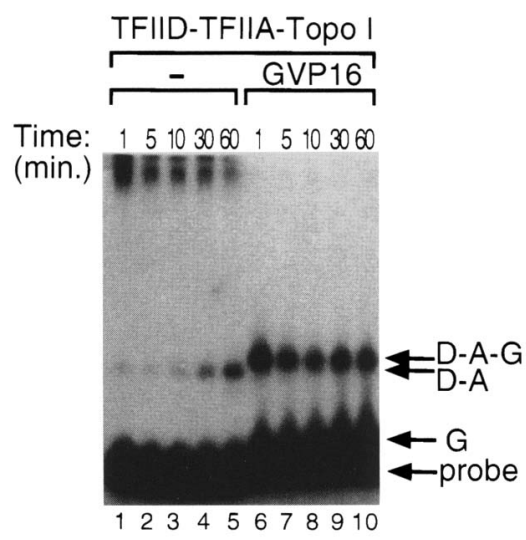

Figure 5. Activator increases the rate of formation of the TFIID-TFIIA complex in the presence of topo I. Agarose (1.5\%) gel shifts performed with ${ }^{32} \mathrm{P}$-labeled ML probe with three Gal4binding sites in the presence of $100 \mathrm{ng}$ competitor poly(dGdC). Topo I, TFIID, and TFIIA were incubated with probe in the absence (lanes 1-5) and presence (lanes 6-10) of Gal4-VP16 for the indicated times at $30^{\circ} \mathrm{C}$ and then analyzed by agaroseEMSA. Gel was dried down and subjected to autoradiography.

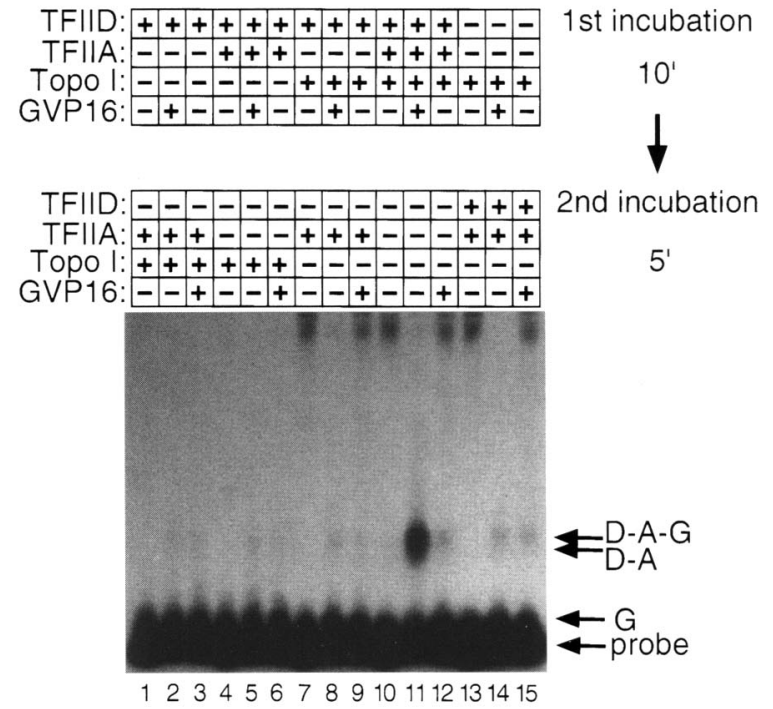

Figure 6. Order of addition demonstrates the requirement for concomitant presence of TFIID, TFIIA, activator, topo I, and promoter for enhanced complex formation. Agarose $(1.5 \%)$ gel shifts performed with ${ }^{32} \mathrm{P}$-labeled ML probe with three Gal4binding sites in the presence of $100 \mathrm{ng}$ of competitor poly(dGdC). Factors indicated were incubated for $10 \mathrm{~min}$ at $30^{\circ} \mathrm{C}$ with probe. The second incubation, with factors indicated, for 5 minutes at $30^{\circ} \mathrm{C}$ preceded analysis by agarose-EMSA. The gel was dried down and subjected to autoradiography.

produced little complex when TFIIA was added during the second incubation, without respect to activator. However, when all four factors were present in the first incubation with probe, rapid stimulation of complex formation was observed (lane 11). The inclusion of topo I with probe in the first incubation followed by TFIID and TFIIA produced little complex with or without activator (lanes 13-15). Thus, the enhanced rate of TFIID-TFIIA complex formation by topo I and activator showed similar phenomenology to the transcription order-of-addition experiments-depending on the concomitant incubation of all four factors with the probe. This suggests that a highly specific cointeraction of the factors with the promoter is required for enhanced complex formation.

\section{Mutant topo I coactivates and stimulates complex formation}

A catalytically inactive point mutant topo I has been described that retains its DNA-binding properties but does not relax supercoiled DNA (Madden and Champoux 1992) This protein has been shown to coactivate transcription indistinguishably from the wild-type protein in vitro, in a less well-defined transcription system (Merino et al. 1993). To examine the functional requirements of topo I for coactivation and enhancement of TFIID-TFIIA complex formation, the mutant enzyme was tested in the transcription assay and in EMSA. In Figure 7, mutant topo I was titrated into transcription reactions in the absence (lanes 1-6) and in the presence (lanes 7-12) of 
activator. Similarly to the wild-type protein, the mutant protein boosted and then repressed basal transcription (lanes 1-6). However, in the presence of activator, the mutant protein potentiated stimulation of transcription on the ML-3G template, but the majority of transcripts failed to elongate to the end of the 390-nucleotide G-less cassette (lanes 7-12). The generation of these short transcription products was not attributable to the presence of contaminating nucleases in the mutant topo I preparation (data not shown). Furthermore, addition of less pure TFIIA to reactions containing mutant topo I generated complete length transcripts and coactivation patterns identical to those for the wild-type protein (data not shown). We assume that Merino et al. (1993), who first identified coactivation with mutant topo I, had components in their reaction that facilitated efficient elongation. These results suggest that the binding properties of topo I are sufficient for coactivation while the relaxing activity may be required for efficient elongation.

Similarly to the wild-type protein, the mutant topo I dramatically enhanced formation of the TFIID-TFIIA complex in the presence of activator (Fig. 8). In the absence of Gal4-VP16 (lanes 1-4), little TFIID-TFIIA complex was generated upon mutant topo I titration during the 20-min binding reaction. However, in the presence of activator (lanes 5-8), titration of the mutant topo I dramatically stimulated and then repressed TFIID-TFIIA complex formation. Thus, the mutant topo I protein stimulates formation of the TFIID-TFIIA complex in the presence of activator similarly to the wild-type protein, indicating that the ability to relax supercoiled DNA is not required for this phenomenon (see Discussion).

\section{Footprint of activated TFIID-TFIIA complex}

To confirm and extend the observation that topo I facilitates the formation of the TFIID-TFIIA complex, DNase I footprinting was used to examine the effect in solution

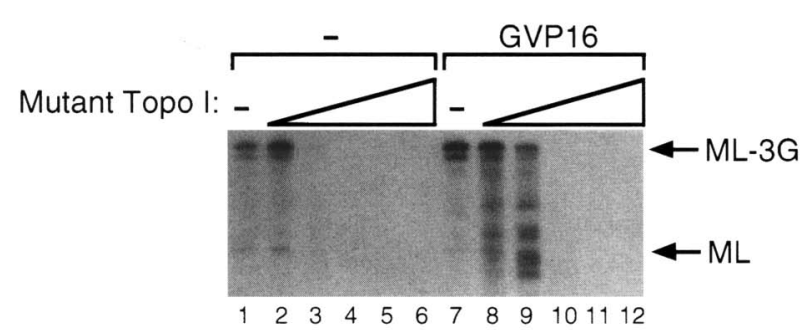

Figure 7. Catalytically inactive point mutant topo I potentiates activation with Gal4-VP16 but does not support efficient elongation. ML transcript ( 180 nucleotides) was generated by 25 ng of supercoiled MLP basal control promoter. ML-3G transcript (390 nucleotides) was generated by $25 \mathrm{ng}$ of supercoiled MLP with three Gal4-binding sites fused upstream. Mutant topo I was titrated $(100,200,300,400,500 \mathrm{ng})$ in the absence (lanes 1-6) and presence (lanes 7-12) of the activator Gal4-VP16. Transcription reactions were analyzed, after purification, on $10 \%$ polyacrylamide gels with $8 \mathrm{M}$ urea, and subjected to autoradiography.

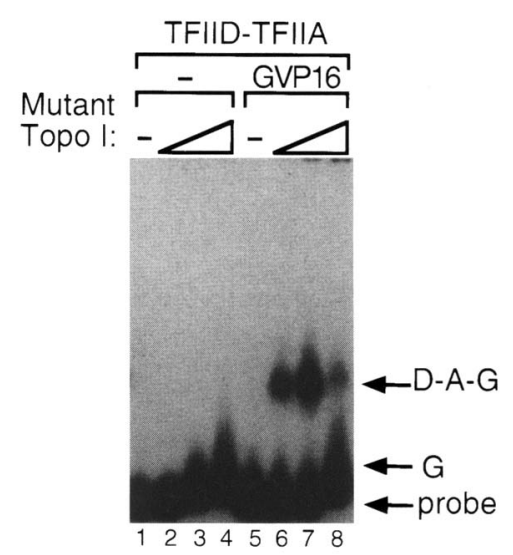

Figure 8. Catalytically inactive point mutant topo I potentiates formation of the TFIID-TFIIA complex in the presence of activator. Agarose (1.5\%) gel shifts performed with ${ }^{32}$ P-labeled ML probe with three Gal4-binding sites in the presence of 100 ng of competitor poly(dGdC). Mutant topo I was titrated (100, $200,400 \mathrm{ng}$ ) in the absence (lanes 1-4) and presence (lanes 5-8) of Gal4-VP16 and incubated for $20 \mathrm{~min}$ at $30^{\circ} \mathrm{C}$ prior to analysis by agarose-EMSA. The gel was dried down and subjected to autoradiography.

(Fig. 9). Under conditions identical to those used in the EMSA reactions, complexes were incubated for $30 \mathrm{~min}$ and then subjected to DNase I cleavage. Topo I was included in the even-numbered lanes, and activator was added in alternate pairs of lanes. Topo I alone did not protect sequences in the promoter region (lane 2). Upon addition of saturating amounts of Gal4-VP16, the three Gal4 sites were protected and remained unaltered by the addition of topo I (lanes 3,4$)$. No protection of the TATA region was detected upon addition of TFIID in the absence or presence of activator and topo I (lanes 5-8). Slight protection of the TATA region was observed when TFIID, TFIIA, and topo I were incubated with the probe (lane 10). Dramatically, complete protection of the TATA region, a hypersensitive site at -43 , and an extended footprint of protection to approximately +37 was observed when TFIID, TFIIA, topo I, and activator were all included in the reaction (lane 12). Thus, DNase I footprinting confirms the results of the EMSA analysis wherein formation of the TFIID-TFIIA complex was strongly stimulated by topo $I$ in the presence of activator.

\section{Presence of topo I on the promoter}

Topo I was not detected on the promoter by DNase I footprinting (Fig. 9), although stoichiometric amounts of protein were present. To further examine the mechanism of coactivation by topo I, a self-footprinting assay with the drug camptothecin was used to determine whether topo I was present in the initiation complex on the promoter. In the presence of this drug, a covalent topo I-3'-DNA intermediate is trapped, generating a nick in the DNA at the site of chemistry (Hsiang et al. 1985). Using an end-labeled probe, the sites of topo I binding 


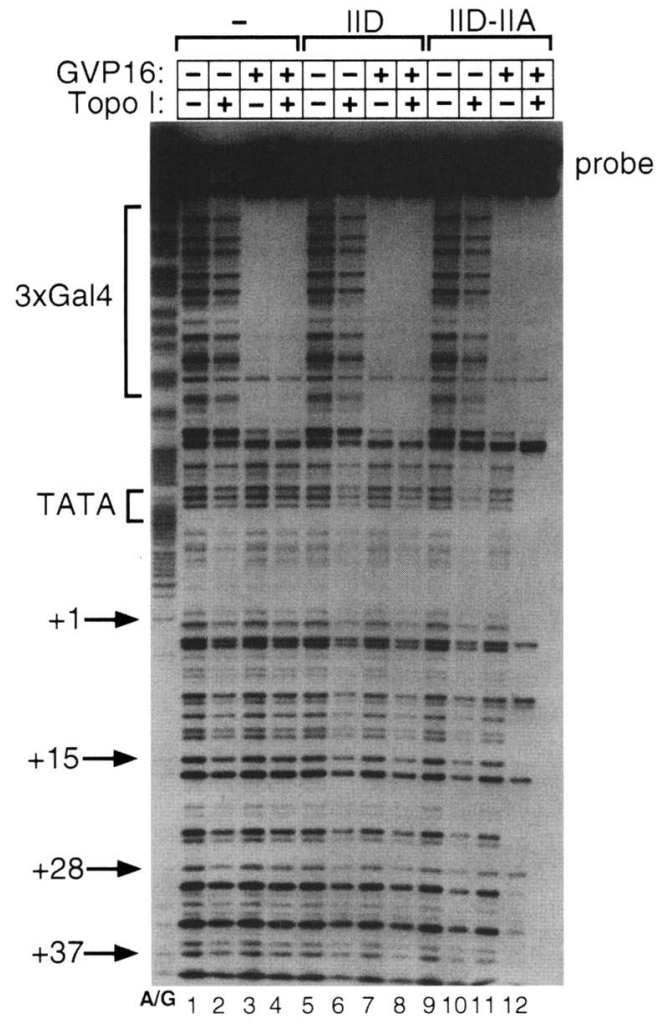

Figure 9. DNase I footprinting demonstrates recruitment of the TFIID-TFIIA complex to the promoter by activator and coactivating topo I. Complexes were formed as in EMSA reactions and then subjected to DNase I cleavage prior to analysis on $8 \%$ polyacrylamide gels- $8 \mathrm{M}$ urea gels. The dried-down gel was subjected to autoradiography.

(i.e., nicking) can be determined accurately. Complexes were formed under transcription conditions in the presence of $25 \mu \mathrm{M}$ camptothecin, and the reactions were subsequently processed with proteinase $\mathrm{K}$ and SDS, and analyzed by denaturing PAGE (see Materials and Methods). At topo I levels that strongly activated transcription and facilitated formation of the TFIID-TFIIA complex in the presence of activator, nicks were generated in the promoter region at several sites both upstream of the TATA box at $-40,-45,-80$ (and farther upstream near the terminus of the probe), relative to the start site of transcription, and downstream at -25 and -4 (Fig. 10, lane 8 ). This pattern was not altered by the addition of TFIID or TFIIA (data not shown). At fivefold lower topo I concentrations, where coactivation and complex facilitation were manifested less strongly, no nicks were generated on the probe (lane 2). This dramatic decrease in the frequency of nicks by topo I, with a fivefold change in concentration, probably reflects the cooperative binding of the protein. However, the addition of TFIID (lane 3) to the binding reaction with this low level of topo I regenerated a pattern of major nicks (lane 8). TBP was unable to regenerate this cleavage pattern with topo I (lane 4) nor did TFIIA have any effect with TFIID, TBP, or alone (data not shown). The addition of activator alone to topo I and probe did not generate nicks (lane 5), nor did activator significantly alter the pattern of cleavage sites in the presence of TFIID (lane 6), TBP (lane 7), or activator plus TFIIA (data not shown). The nicking obtained upon coincubation of topo I with TFIID was not attributable to contaminating topo I as TFIID on its own did not generate any nicks (data not shown). Furthermore, no topo I was detected in the TFIID preparation by both Western analysis and DNA relaxation assay (data not shown). The topo I nicking sites identified are in regions of the promoter protected from cleavage by DNase I by both TFIID and Gal4-VP16. Thus, at concentrations where the TFIID-TFIIA complex is strongly facilitated, topo I is present on the promoter region at multiple sites. At lower concentrations of topo I, an association of TFIID and topo I with the promoter (in a TAF-dependent manner) is detectable, suggesting that the coactivator functions locally at the site of formation of the TFIID-TFIIA complex and further implying a role for the TAFs in interacting with the coactivator during the activation process.

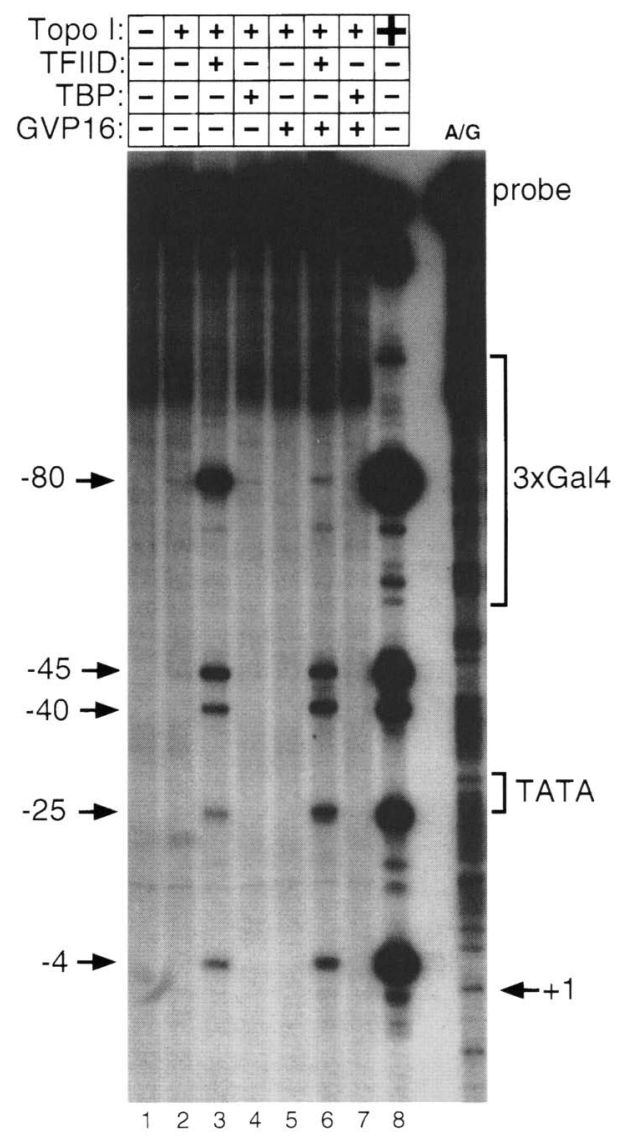

Figure 10. Camptothecin-mediated cleavage of the ML-3G promoter region by topo I. Complexes were formed under gelshift conditions in the presence of $25 \mu \mathrm{M}$ camptothecin. The probe was subsequently processed as described (see Materials and Methods/ and analyzed by PAGE-urea. The dried-down gel was subjected to autoradiography. 


\section{Discussion}

Formation of the TFIID-TFIIA complex is a critical first step in activation potentiated by topo $I$. This activation was dependent on the $\mathrm{TAF}_{\mathrm{II}}$ coactivators as is activation in nuclear extracts. Maximal activation required the concomitant presence of TFIID, TFIIA, topo I, activator, and promoter and correlated with a dramatic increase in the rate and extent of formation of the TFIID-TFIIA complex as detected both by electrophoresis in agarose gels and by DNase I footprinting. Enhanced TFIID-TFIIA complex formation was also dependent on $\mathrm{TAF}_{\mathrm{II}} \mathrm{s}$ and the concomitant reaction of all four factors. A catalytically inactive point mutant of topo I-mediated stimulation of initiation and correspondingly enhanced TFIIDTFIIA complex formation. The ability of camptothecin to stabilize the topo I-nicked DNA intermediate was used to show that the coactivator could be recruited to the promoter region by the TFIID complex, dependent on $\mathrm{TAF}_{\mathrm{II}} \mathrm{s}$. Thus, activation potentiated by topo I is associated with an enhanced recruitment of the TFIID complex, dependent on TFIIA and activator.

Both types of order-of-addition experiments, transcription and formation of DNA-binding complexes, suggest that the action of the activator and the coactivator cannot be temporally separated for maximal activation. Specifically, TFIID and TFIIA cannot first be incubated with the promoter, either with activator or coactivator if maximal activation is to occur. A simple recruitment model would suggest that the order of addition would be irrelevant, as TFIID and TFIIA should remain in solution unbound until recruited by activator in the context of the coactivator. There are several possible explanations for this phenomenon. First, without the influence of the activator and coactivator directing it to the initiation site, the TFIID-TFIIA complex could bind DNA tightly with little specificity such that very little is active at the promoter. This is unlikely, as complexes were not observed by EMSA in the absence of activator and coactivator. Another possibility is that in the absence of activator and coactivator, a stable, low activity TFIID-TFIIA complex is slowly formed on the promoter. The conformation of a majority of these complexes would not mediate highly active transcription. Consistent with this hypothesis, the study of coactivation by HMG proteins suggested that once bound, TFIID was not amenable to activation (Shykind et al. 1995). Furthermore, analysis of open complex formation by permanganate footprinting demonstrated a similar refractivity to activation when TFIID bound alone (Wang et al. 1992). Thus, it is likely that simultaneous interaction of all four factors produces a structural change in the DNA-bound TFIID-TFIIA complex important for the activated state. Generation of this complex probably requires the precisely choreographed formation of a stereospecific complex involving TFIID, TFIIA, activator, topo I, and promoter.

The TFIID-TFIIA complex has been reported to be an early, critical stage of activation (Wang et al. 1992; Lieberman and Berk 1994; Kaiser et al. 1995; Shykind et al. 1995), and TFIIA has been shown to be required for activation (Ma et al. 1993; Ozer et al. 1994; Sun et al. 1994). Our results are consistent with and extend these findings. They also explain the results of Wang et al. (1992), who observed a decrease in the lag time of open complex formation, as assayed by permanganate footprinting, when partially purified TFIID and TFIIA were coincubated with the adenovirus $\mathrm{E} 4$ promoter and Gal4$\mathrm{AH}$. It is almost certain that the TFIID fraction used by Wang et al. (1992) contained topo I, as TFIID and topo I coimmunoprecipitate from such fractions (Merino et al. 1993). Lieberman and Berk (1994) demonstrated that the formation of the TFIID-TFIIA complex on the E4 promoter with seven upstream Zta sites was greatly enhanced by the Zta activator (similarly demonstrated and extended by Chi et al. 1995). Here, we have demonstrated a similar dramatic enhancement of TFIID-TFIIA complex formation, completely dependent on the presence of topo I, which alone coactivated strong stimulation in the system. The requirement of coactivator for rapid complex formation in our system may be attributable to the smaller number of activator binding sites (three) compared to that of Lieberman and Berk (seven) and the nature of the activators used /Gal4-VP16 vs. $\mathrm{Zta}$. However, whereas Lieberman and Berk required a poorly defined coactivator fraction to attain stimulation, activation in the system used here was achieved with defined components.

It is likely that no single activator-basal factor or activator-coactivator interaction will account for activation of Pol II transcription. Individual binding steps may become limiting in the context of different core promoter elements (Das et al. 1995) or in the context of different chromatin structures. Activators may have different activities in the presence of different coactivators (Lehming et al. 1994), and different coactivators may require different core promoter setups (Shykind et al. 1995). Additionally, the activation process is dynamic and must be viewed as a tightly choreographed series of steps such that individual interactions may only become rate limiting under particular conditions. Increasingly, factors that effect transcription are being identified that interact not only with the protein components of the reaction but also bind and potentially alter the conformation of DNA in the promoter region. Such factors include the nonhistone chromosomal proteins of the three HMG families /Thanos and Maniatis 1992; Paranjape et al. 1995; Shykind et al. 1995), an HMG box containing factors such as upstream binding factor (UBF) and lymphoid enhancer-binding factor 1 (LEF-1) (Jantzen et al. 1990; Giese et al. 1992), and proteins with DNA-binding activity like PC-4 (Ge and Roeder 1994; Kretzschmar et al. 1994). Topo I binds to DNA, preferring irregular structures such as nodes where DNA strands cross over each other (Zechiedrich and Osheroff 1990). Furthermore, bent DNA has been reported to stabilize topo I binding (Krogh et al. 1991). Thus, topo I probably shares the common property of being an architectural factor, stabilizing bent or distorted DNA structure upon binding. In part, its function as a coactivator is probably the result of these properties. 
Merino and colleagues reported the ability of topo I to associate through protein-protein interactions with the TFIID complex (Merino et al. 1993). In this study camptothecin-mediated cleavage has demonstrated the ability of topo I to bind to the promoter, in the region of the promoter bound by TFIID. Additionally, at lower concentrations, TFIID can recruit topo I, via the $\mathrm{TAF}_{\mathrm{II}} \mathrm{s}$, to several sites in the promoter region. Thus, it is likely that topo I is present with TFIID, TFIIA, and activator on the promoter. In this model, topo I facilitates the recruitment of the TFIID complex by activator, perhaps molds the structure of the TFIID complex, dependent on TFIIA, and probably is positioned on the promoter with TFIID. In this way, topo I would be loaded into the initiation complex where it can facilitate the unwinding of DNA that is necessary for the movement of the template relative to the polymerase during transcription elongation (Wang 1985). Topo I is essential for viability in Drosophila (Lee et al. 1993) and could be critical for transcription by Pol II. However, the ability of Saccharomyces cerevisiae to survive without topo I suggests that the transcription machinery in yeast is not uniquely dependent on this protein.

Thus, a model of early activation can be formulated wherein topo I mediates a protein-DNA structure with TFIID such that an active initiation complex is rapidly generated in the presence of activator and TFIIA. This activation event is characterized by an increase in the rate and extent of formation of an active TFIID/TFIIA/ activator/coactivator complex. A manifestation of this activation may be an increased recruitment of TFIIB, TFIIF, and RNA Pol II (Choy and Green 1993). This early activated complex could also be a high affinity platform for a holopolymerase complex (Kim et al. 1994; Koleske and Young 1994).

\section{Materials and methods}

\section{Purification of topo I}

Human DNA topo I was purified from HeLa cells as in Stewart et al. (1996). Three liters of cells at $1 \times 10^{6} \mathrm{cells} / \mathrm{ml}$ were washed twice in $4^{\circ} \mathrm{C}$ PBS and lysed in $40 \mathrm{ml}$ of lysis buffer $150 \mathrm{mM} \mathrm{KCl}$, $10 \mathrm{~mm}$ Tris- $\mathrm{HCl}$ at $\mathrm{pH} 7.5,2 \mathrm{~mm} \mathrm{MgCl}, 1 \%$ Triton X-100, 15 $\mathrm{mM} \mathrm{DTT}, 0.15 \mathrm{mg} / \mathrm{ml}$ of PMSF, $0.05 \mathrm{mg} / \mathrm{ml}$ of aprotinin) by shaking. Nuclei were pelleted by centrifugation and washed in $40 \mathrm{ml}$ of lysis buffer without Triton X-100. Nuclei were repelleted by centrifugation and resuspended in $10 \mathrm{ml}$ of lysis buffer without Triton X-100. Fifteen milliliters of extraction buffer $(2$ $\mathrm{M} \mathrm{NaCl}, 80 \mathrm{~mm}$ Tris- $\mathrm{HCl}$ at $\mathrm{pH} 7.5,20 \%$ glycerol, 2 mM EDTA) was added, and the lysis was mixed with a magnetic stir bar while $15 \mathrm{ml}$ of PEG buffer (18\% PEG-8000, $1 \mathrm{M} \mathrm{NaCl}, 10 \%$ glycerol) was added dropwise. Stirring was continued for $1 \mathrm{hr}$ at $4^{\circ} \mathrm{C}$. The lysate was cleared by centrifugation of $10,000 \mathrm{~g}$ for 10 min, and the pellet was discarded. The supernatant was dialyzed overnight against 2 liters of potassium phosphate buffer $(250$ $\mathrm{mM} \mathrm{KPO}, 10 \%$ glycerol, $1 \mathrm{~mm}$ DTT, $1 \mathrm{~mm}$ EDTA, $0.1 \mathrm{~mm}$ PMSF). The dialysate was cleared by centrifugation and flowed over a $5-\mathrm{ml}$ bed of phenyl-Superose (Pharmacia) equilibrated with $250 \mathrm{mM} \mathrm{KPO}_{4}$ buffer (pH 7.4) (with $1 \mathrm{~mm}$ DTT, $1 \mathrm{~mm}$ EDTA, $0.1 \mathrm{~mm}$ PMSF) and onto a 5-ml bed of phosphocellulose P11 (Whatman) equilibrated with $250 \mathrm{~mm} \mathrm{KPO}_{4}$ buffer. The phosphocellulose column was washed with $250 \mathrm{~mm} \mathrm{KPO}_{4}$ buffer and eluted with $700 \mathrm{mM} \mathrm{KPO}_{4}$ buffer. The eluate was dialyzed against $100 \mathrm{mM} \mathrm{KPO}{ }_{4}$ buffer and then loaded on a mono-S FPLC (Pharmacia) column and developed with a linear gradient of $\mathrm{KPO}_{4}$ buffer $(100-500 \mathrm{~mm})$. The topo I that eluted around $150 \mathrm{mM} \mathrm{KPO}_{4}$ was dialyzed down to $100 \mathrm{mM} \mathrm{KPO}_{4}$ and batch-cleared with $2 \mathrm{ml}$ of mono-Q resin (Pharmacia) for $1 \mathrm{hr}$. Finally, the pure topo I was dialyzed against $50 \%$ glycerol, 10 $\mathrm{mm}$ Tris- $\mathrm{HCl}(\mathrm{pH} 7.5), 1 \mathrm{~mm}$ DTT, and $1 \mathrm{~mm}$ EDTA and stored at $-20^{\circ} \mathrm{C}$. Topo I obtained from commercial sources had indistinguishable activities in the transcription assays.

\section{In vitro transcription}

Transcription reagents and reactions were performed as described (Shykind et al. 1995). Briefly, 10 ng of HA-tagged HeLa eTFIID (Zhou et al. 1992) or $10 \mathrm{ng}$ of rTBP, $5 \mathrm{ng}$ HeLa TFIIA purified to homogeneity, $20 \mathrm{ng}$ of rTFIIB, $4 \mathrm{ng}$ of rTFIIE ( $3 \mathrm{ng}$ of p56+1 ng of p34), $60 \mathrm{ng}$ of rTFIIF (50 ng of RAP30+10 ng of RAP74), 50 ng of RNA Pol II prepared either from calf thymus (Parvin et al. 1994) or from chinese hamster ovary (CHO) cells (Carthew et al. 1988), purified HeLa TFIIH (Shykind et al. 1995) and $10 \mathrm{ng}$ of Gal4-VP16 were incubated with a total of $50 \mathrm{ng}$ of supercoiled DNA template in $0.02 \mathrm{M}$ HEPES-NaOH $(\mathrm{pH} 7.9)$, $20 \%$ glycerol, $0.001 \mathrm{M}$ EDTA, $0.060 \mathrm{M} \mathrm{KCl}, 0.001 \mathrm{M}$ dithiothreitol, $0.005 \mathrm{M} \mathrm{MgCl}_{2}, 0.05 \% \mathrm{NP}-40$, in $20-\mu \mathrm{l}$ reactions at $30^{\circ} \mathrm{C}$ for $60 \mathrm{~min}$ or for the times indicated.

\section{EMSA and DNase I footprinting}

Agarose gel shifts and DNase I footprinting reactions were performed as described (Shykind et al. 1995). Briefly, protein-DNA binding reactions were done following published protocol $(\mathrm{Li}$ eberman and Berk 1994), with several modifications. Binding was done in $20 \mu \mathrm{l}$ of binding buffer (12.5 mM HEPES at $\mathrm{pH} 7.9$, $12.5 \%$ glycerol, $5 \mathrm{~mm}$ EDTA, $70 \mathrm{~mm} \mathrm{KCl}, 1 \mathrm{~mm} \mathrm{DTT}, 0.5 \mathrm{mg} /$ $\mathrm{ml}$ of BSA, $5 \mu \mathrm{g} / \mathrm{ml}$ of poly[d(G-C)], and $1 \mathrm{ng}$ of probe) at $30^{\circ} \mathrm{C}$ for the times indicated. Gels for EMSA were cast with $1.5 \%$ agarose in $0.5 \times \mathrm{TBE}$ with $5 \mathrm{~mm} \mathrm{MgCl}_{2}$. Gels were run in $0.5 \times$ TBE with $5 \mathrm{mM} \mathrm{MgCl}_{2}$ at $70 \mathrm{~V}$ for $3 \mathrm{hr}$ and then dried onto DE81 paper (Whatman). For the DNase I footprinting assay, 16 $\mu \mathrm{l}$ of DNase I solution containing 1 ng of DNase I/Worthington Enzymes; DPFF grade) and $5 \mathrm{mM} \mathrm{CaCl}_{2}$ were added to the binding reaction above. After $1 \mathrm{~min}$ of incubation at room temperature, $90 \mu \mathrm{l}$ of stop solution (20 mM EDTA at $\mathrm{pH} 8.0,1 \%$ SDS, $200 \mathrm{~mm} \mathrm{NaCl}, 250 \mu \mathrm{g} / \mathrm{ml}$ of yeast tRNA) was added. The DNase I digestion was analyzed by sequencing gel electrophoresis.

\section{Camptothecin cleavage assay}

Binding reactions were performed as in footprint reaction and EMSA, with the addition of camptothecin (Sigma, $10 \mathrm{~mm}$ in DMSO) to $25 \mu \mathrm{M}$ in the reactions from $0 \mathrm{~min}$. After reaction at $30^{\circ} \mathrm{C}$ for $30 \mathrm{~min}$, SDS was added to $0.2 \%$ and proteinase $\mathrm{K}$ was added to a final concentration of $50 \mu \mathrm{g} / \mathrm{ml}$. Reactions were incubated at $37^{\circ} \mathrm{C}$ for $30 \mathrm{~min}$, followed by extraction with phenol $/$ chloroform/isoamyl alcohol and ethanol-acetate precipitation. Finally, reactions were analyzed on $8 \%$ polyacrylamide gel-urea gel, and subjected to autoradiography.

\section{Acknowledgments}

We thank members of the Sharp laboratory for useful discussions and critical reading of early drafts of this manuscript. We also thank R. Issner for technical assistance and M. Siafaca for expert administrative support. This work was supported by U.S. 
Public Health Service grant PO1-CA42063 from the National Cancer Institute, National Institutes of Health (NIH), by cooperative agreement CDR-8803014 from the National Science Foundation to P.A.S., and, partially, by National Cancer Institute Cancer Center Support (core) grant P30-CA14051. J.J.C. was supported by the NIH grant GM49156, and L.S. received support from the American Cancer Society (grant PF-3905).

The publication costs of this article were defrayed in part by payment of page charges. This article must therefore be hereby marked "advertisement" in accordance with 18 USC section 1734 solely to indicate this fact.

\section{Note added in proof}

Recently, Chi and Carey (1996) Genes \& Dev. 10: 2540-2550, described results supporting the hypothesis that activation requires formation of an isomerized TFIID-TFIIA complex on the promoter with activator. These results are in agreement with the phenomenology of the data presented here.

\section{References}

Bendixen, C., B. Thomsen, J. Alsner, and O. Westergaard. 1990. Camptothecin-stabilized topoisomerase I-DNA adducts cause premature termination of transcription. Biochemistry 29: 5613-5619.

Buratowski, S. 1994. The basics of basal transcription by RNA polymerase II. Cell 77: 1-3.

Carthew, R.W., M. Samuels, and P.A. Sharp. 1988. Formation of transcription preinitiation complexes with an amanitin-resistant RNA polymerase II. J. Biol. Chem. 263: 17128-17135

Chi, T., P. Lieberman, K. Ellwood, and M. Carey. 1995. A general mechanism for transcriptional synergy by eukaryotic activators. Nature 377: 254-257.

Choy, B. and M.R. Green. 1993. Eukaryotic activators function during multiple steps of preinitiation complex assembly. Nature 366: 531-536.

Das, G., C.S. Hinkley, and W. Herr. 1995. Basal promoter elements as a selective determinant of transcriptional activator function. Nature 374: 657-660.

Davison, B.L., J.M. Egly, E.R. Mulvihill, and P. Chambon. 1983. Formation of stable preinitiation complexes between eukaryotic class B transcription factors and promoter sequences. Nature 301: 680-686.

Dynlacht, B.D., T. Hoey, and R. Tjian. 1991. Isolation of coactivators associated with the TATA-binding protein that mediate transcriptional activation. Cell 66: 563-576.

Egyhazi, E. and E. Durban. 1987. Microinjection of anti-topoisomerase I immunoglobulin G into nuclei of Chironomus tentans salivary gland cells leads to blockage of transcription elongation. Mol. Cell. Biol. 7: 4308-4316.

Fleischmann, G., G. Pflugfelder, E.K. Steiner, K. Javaherian, G.C. Howard, J.C. Wang, and S.C. Elgin. 1984. Drosophila DNA topoisomerase I is associated with transcriptionally active regions of the genome. Proc. Nat1. Acad. Sci. 81: 6958-6962.

Ge, H. and R.G. Roeder. 1994. Purification, cloning, and characterization of a human coactivator, PC4, that mediates transcriptional activation of class II genes. Cell 78: 513-523.

Giese, K., J. Cox, and R. Grosschedl. 1992. The HMG domain of lymphoid enhancer factor 1 bends DNA and facilitates assembly of functional nucleoprotein structures. Cell 69: 185195.

Gill, G., E. Pascal, Z.H. Tseng, and R. Tjian. 1994. A glutaminerich hydrophobic patch in transcription factor $\mathrm{Spl}$ contacts the dTAFII 10 component of the Drosophila TFIID complex and mediates transcriptional activation. Proc. Natl. Acad. Sci. 91: 192-196.

Gilmour, D.S., G. Pflugfelder, J.C. Wang, and J.T. Lis. 1986. Topoisomerase I interacts with transcribed regions in Drosophila cells. Cell 44: 401-407.

Goodrich, J.A., T. Hoey, C.J. Thut, A. Admon, and R. Tjian. 1993. Drosophila TAFII40 interacts with both a VP16 activation domain and the basal transcription factor TFIIB. Cell 75: 519-530.

Hoey, T., R.O. Weinzierl, G. Gill, J.L. Chen, B.D. Dynlacht, and R. Tjian. 1993. Molecular cloning and functional analysis of Drosophila TAF110 reveal properties expected of coactivators. Cell 72: 247-260.

Hsiang, Y.-H., R. Hertzeberg, S. Hecht, and L.F. Liu. 1985. Camptothecin induces protein-linked DNA breaks via mammalian DNA topoisomerase I. J. Biol. Chem. 260: 14873-14878.

Jantzen, H.M., A. Admon, S.P. Bell, and R. Tjian. 1990. Nucleolar transcription factor hUBF contains a DNA-binding motif with homology to HMG proteins. Nature 344: 830-836.

Kaiser, K., G. Stelzer, and M. Meisterernst. 1995. The coactivator p15 (PC4) initiates transcriptional activation during TFIIA-TFIID-promoter complex formation. EMBO $J$. 14: 3520-3527.

Kim, Y.J., S. Bjorklund, Y. Li, M.H. Sayre, and R.D. Kornberg. 1994. A multiprotein mediator of transcriptional activation and its interaction with the C-terminal repeat domain of RNA polymerase II. Cell 77: 599-608.

Kobayashi, N., T.G. Boyer, and A.J. Berk. 1995. A class of activation domains interacts directly with TFIIA and stimulates TFIIA-TFIID-promoter complex assembly. Mol. Cell. Biol. 11: $6465-6473$.

Koleske, A.J. and R.A. Young. 1994. An RNA polymerase II holoenzyme responsive to activators. Nature 368: 466-469.

Kretzschmar, M., M. Meisterernst, and R.G. Roeder. 1993. Identification of human DNA topoisomerase I as a cofactor for activator-dependent transcription by RNA polymerase II. Proc. Natl. Acad. Sci. 90: 11508-11512.

Kretzschmar, M., K. Kaiser, F. Lottspeich, and M. Meisterernst. 1994. A novel mediator of class II gene transcription with homology to viral immediate-early transcriptional regulators. Cell 78: 525-534.

Krogh, S., U.H. Mortensen, O. Westergaard, and B.J. Bonven. 1991. Eukaryotic topoisomerase I-DNA interaction is stabilized by helix curvature. Nucleic Acids Res. 19: 1235-1241.

Lee, M.P., S.D. Brown, A. Chen, and T.S. Hsieh. 1993. DNA Topoisomerase I is essential in Drosophila melanogaster. Proc. Natl. Acad. Sci. 90: 6656-6660.

Lehming, N., D. Thanos, J.M. Brickman, J. Ma, T. Maniatis, and M. Ptashne. 1994. An HMG-like protein that can switch a transcriptional activator to a repressor. Nature 371: 175 179.

Lieberman, P.M. and A.J. Berk. 1994. A mechanism for TAFs in transcriptional activation: Activation domain enhancement of TFIID-TFIIA-promoter DNA complex formation. Genes \& Dev. 8: 995-1006.

Lin, Y.S. and M.R. Green. 1991. Mechanism of action of an acidic transcriptional activator in vitro. Cell 64: 971-981.

Ma, D., H. Watanabe, F. Mermelstein, A. Admon, K. Oguri, X. Sun, T. Wada, T. Imai, T. Shiroya, D. Reinberg, et al. 1993. Isolation of a cDNA encoding the largest subunit of TFIIA reveals functions important for activated transcription. Genes \& Dev. 7: 2246-2257.

Madden, K.R. and J.J. Champoux. 1992. Overexpression of human topoisomerase I in baby hamster kidney cells: Hyper- 
sensitivity of clonal isolates to camptothecin. Cancer Res. 52: $525-532$.

'Meisterernst, M., A.L. Roy, H.M. Lieu, and R.G. Roeder. 1991. Activation of class II gene transcription by regulatory factors is potentiated by a novel activity. Cell 66: 981-993.

Merino, A., K. Madden, W. Lane, J. Champoux, and D. Reinberg. 1993. DNA topoisomerase I is involved in both repression and activation of transcription. Nature 365: 221-232.

Nikolov, D.B., S.H. Hu, J. Lin, A. Gasch, A. Hoffmann, M. Horikoshi, N.H. Chua, R.G. Roeder, and S.K. Burley. 1992. Crystal structure of TFIID TATA-box binding protein. Nature 360: 40-46.

Ozer, J., P.A. Moore, A.H. Bolden, A. Lee, C.A. Rosen, and P.M. Lieberman. 1994. Molecular cloning of the small $(\gamma)$ subunit of human TFIIA reveals functions critical for activated transcription. Genes \& Dev. 8: 2324-2335.

Paranjape, S.M., A. Krumm, and J.T. Kadonaga. 1995. HMG17 is a chromatin-specific transcriptional coactivator that increases the efficiency of transcription initiation. Genes \& Dev. 9: 1978-1991.

Parvin, J.D., B.M. Shykind, R.E. Meyers, J. Kim, and P.A. Sharp. 1994. Multiple sets of basal factors initiate transcription by RNA polymerase II. J. Biol. Chem. 269: 18414-18421.

Roberts, S.G.E. and M.R. Green. 1994. Activator-induced conformational change in general transcription factor TFIIB. $\mathrm{Na}$ ture 371: 717-720.

Samuels, M., A. Fire, and P.A. Sharp. 1982. Separation and characterization of factors mediating accurate transcription by RNA polymerase II. J. Biol. Chem. 257: 14419-14427.

Sauer, F., S.K. Hansen, and R. Tjian. 1995. Multiple TAFIIs directing synergistic activation of transcription. Science 270: $1783-1788$.

Sawadogo, M. and R.G. Roeder. 1985. Interaction of a genespecific transcription factor with the adenovirus major late promoter upstream of the TATA box region. Cell 43: 165175.

Shykind, B.M., J. Kim, and P.A. Sharp. 1995. Activation of the TFIID-TFIIA complex with HMG-2. Genes \& Dev. 9: 13541365.

Stewart, A.F. and G. Schutz. 1987. Camptothecin-induced in vivo topoisomerase I cleavages in the transcriptionally active tyrosine aminotransferase gene. Cell 50: 1109-1117.

Stewart, A.F., R.E. Herrera, and A. Nordheim. 1990. Rapid induction of $\mathrm{c}$-fos transcription reveals quantitative linkage of RNA polymerase II and DNA topoisomerase I enzyme activities. Cell 60: 141-149.

Stewart, L., G.C. Ireton, L.H. Parker, K.R. Madden, and J.J. Champoux. 1996. Biochemical and biophysical analyses of recombinant forms of human topoisomerase I. J. Biol. Chem. 271: 7593-7601.

Sun, X., D. Ma, M. Sheldon, K. Yeung, and D. Reinberg. 1994. Reconstitution of human TFIIA activity from recombinant polypeptides: A role in TFIID-mediated transcription. Genes \& Dev. 8: $2336-2348$.

Thanos, D. and T. Maniatis. 1992. The high mobility group protein $H M G \mathrm{I}(\mathrm{Y})$ is required for NF- $\mathrm{k}$-dependent virus induction of the human IFN- $\beta$ gene. Cell 71: 777-789.

Tjian, R. and T. Maniatis. 1994. Transcriptional activation: A complex puzzle with few easy pieces. Cell 77: 5-8.

Verrijzer, C.P., K. Yokimori, J.L. Chen, and R. Tjian. 1994. Drosophila $\mathrm{TAF}_{\mathrm{II}}$ 150: Similarity to yeast TSM-1 and specific binding to core promoter DNA. Science 264: 933-941.

Wang, J.C. 1985. DNA topoisomerases. Annu. Rev. Biochem. 54: 665-697.

- 1992. Template topology and transcription. In Transcriptional regulation (ed. S.L. McKnight and K.R. Yama- moto), pp. 1253-1269. Cold Spring Harbor Laboratory Press, Cold Spring Harbor, NY.

Wang, W., J.D. Gralla, and M. Carey. 1992. The acidic activator GAL4-AH can stimulate polymerase II transcription by promoting assembly of a closed complex requiring TFIID and TFIIA. Genes \& Dev. 6: 1716-1727.

Weisbrod, S.T. 1982. Properties of active nucleosomes as revealed by HMG 14 and 17 chromatography. Nucleic Acids Res. 10: 2017-2042.

White, J., C. Brou, J. Wu, Y. Lutz, V. Moncollin, and P. Chambon. 1992. The acidic transcriptional activator GAL-VP16 acts on preformed template-committed complexes. EMBO $T$. 11: $2229-2240$.

Zechiedrich, E.L. and N. Osheroff. 1990. Eukaryotic topoisomerases recognize nucleic acid topology by preferentially interacting with DNA crossovers. EMBO I. 13: 4555-4562.

Zhang, H., J.C. Wang, and L.F. Liu. 1988. Involvement of DNA topoisomerase I in transcription of human ribosomal RNA genes. Proc. Natl. Acad. Sci. 85: 1060-1064.

Zhou, Q., P.M. Lieberman, T.G. Boyer, and A.J. Berk. 1992. Holo-TFIID supports transcriptional stimulation by diverse activators and from a TATA-less promoter. Genes \& Dev. 6: 1964-1974. 


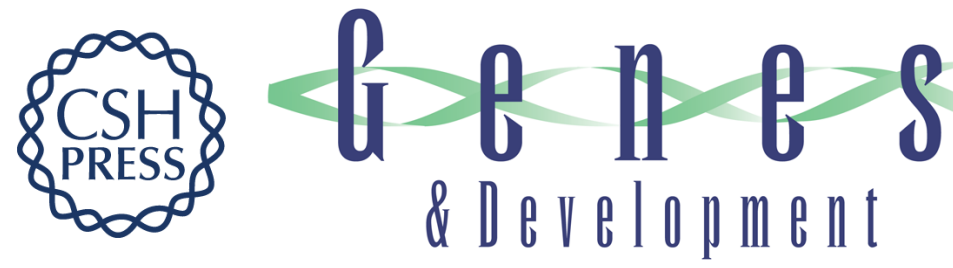

\section{Topoisomerase I enhances TFIID-TFIIA complex assembly during activation of transcription.}

B M Shykind, J Kim, L Stewart, et al.

Genes Dev. 1997, 11:

Access the most recent version at doi:10.1101/gad.11.3.397

References This article cites 56 articles, 22 of which can be accessed free at: http://genesdev.cshlp.org/content/11/3/397.full.html\#ref-list-1

License

Email Alerting

Service

Receive free email alerts when new articles cite this article - sign up in the box at the top right corner of the article or click here.

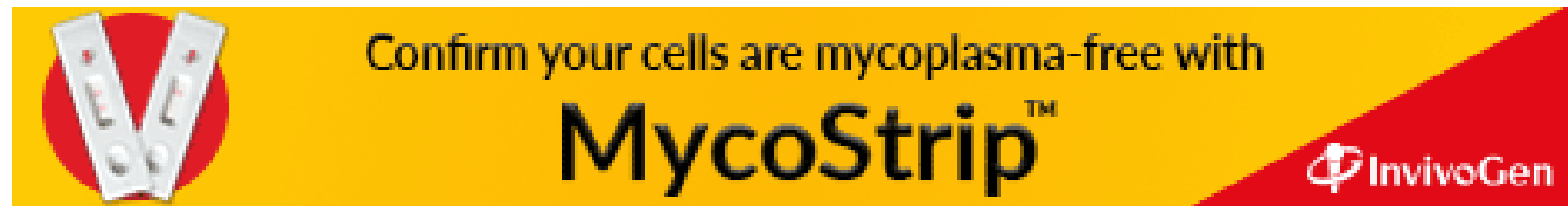

